Introduction: Major injury incidents in underground metalliferous and mineral mines are rare, but if, e.g., a major fire would occur, it is the emergency medical service (EMS) together with the mining company and rescue service who perform the rescue operation. Therefore it is important to develop safe and efficient rescue operation procedures for all the organizations involved, especially the EMS personnel.

Aim: To examine EMS personnel's perceptions and experiences regarding underground mining incidents.

Method: Individual interviews were performed with 13 Swedish EMS personnel. The interviews were transcribed verbatim and analyzed with qualitative content analysis.

Results: The theme "providing the same care in a difficult environment" emerged. Depending on the type of incident, the EMS personnel considered if the injured mining workers could be cared for either outside or in the mine in order to access and care for the injured mining workers as quickly as possible. The EMS personnel mentioned that it was difficult to make the decision if they should enter the mine or not due to the uncertainty of their safety. They also considered that it could be harder to accomplish the same level of care as in other incidents due to the difficult environment. In some instances, they cannot drive their ambulance vehicles into the mine, so they have to prioritize which equipment to bring as well as consider how to transport the patients.

Discussion: The results identify some of the difficulties the EMS find challenging. Therefore the results could be used in finding solutions and making the EMS prepared for an effective and timely response for injured in underground mines.

Prehosp Disaster Med 2019;34(Suppl. 1):s163-s164

doi:10.1017/S1049023X19003728

\section{Research on Design of a Disaster Medicine Course for Clinical Medical Student}

Ms. Zhi Wan, Dr. Hai Hu

West China Hospital of Sichuan University, Chengdu City, China

Introduction: Currently, there is no uniform and standard disaster medicine course for students in medical school.

Aim: To design a disaster medicine course model based on knowledge structure, teaching and evaluation methods according to experts' advice and interest of undergraduates majoring in clinical medicine.

Methods: The first and second level catalog defined as chapters and sections for the disaster medicine curriculum were drafted based on literature and summary of fragmentary experience. The teaching syllabus with methods of teaching and evaluation was initially outlined. The expert consultation form and student questionnaire were designed and validated. Experts in disaster medicine in China were consulted and students in our medical school were investigated. Delphi Methods was used and the chapters and sections were adjusted and weighed according to experts' advice through the Analytic Hierarchy Process. The teaching and evaluation methods for each knowledge module were obtained based on suggestions from experts and students.

Results: A total of 31 experts were consulted. 320 students were inquired. By two rounds of consultation with Kendall coordination coefficient $\mathrm{W}$ value 0.207 , chi-square value 128.781 $(\mathrm{p}=0.01)$, consensus about the knowledge structure for the curriculum were achieved, which consisted of 6 chapters (as Introduction to disaster medicine, incident command, medical knowledge and skills in disaster, public health, ethics in disaster, information management, with the weights of 0.1486 , $0.1999,0.4209,0.0785,0.0748,0.0774$ respectively)and 25 sections. Teaching methods for different knowledge module were determined, which included lecture, demonstration, case discussion, drill and sand table simulation. And the evaluation methods were affirmed as a quiz, written examination, skill test and teamwork test assessed by intra-group and inter-group evaluation.

Discussion: Through scientific investigation of experts in disaster rescue and undergraduates majoring in clinical medicine, a disaster medicine course model for clinical medical students was established.

Prehosp Disaster Med 2019;34(Suppl. 1):s164

doi:10.1017/S1049023X1900373X

\section{Resilience Training of Regional Medical Command and Control \\ RN Jenny Pettersson ${ }^{1}$, Carl-Oscar Jonson $P h D^{1}$, Peter Berggren PhD ${ }^{1}$, Rogier Woltjer $P h D^{2}$, \\ Jonas Hermelin $M S c^{2}$, Erik Prytz $P h D^{1}$}

1. Center for disaster medicine and traumatology, and Department of Clinical and Experimental Medicine, Linköping University, Linköping, Sweden

2. Swedish Defence Research Agency, Stockholm, Sweden

Introduction: Resilience is often described as a desirable holistic approach to disaster preparedness. However, the term has a wide variety of meanings and is hard to operationalize and implement in disaster management. A goal for the EU H2020 project DARWIN was to operationalize resilience for incident management teams.

Aim: To test the resilience operationalization by analyzing command team behaviors in a major incident exercise and trace observations to resilience theory.

Methods: A regional medical command and control team $(\mathrm{n}=11)$ was observed when performing in a functional simulation exercise of a mass casualty incident (300 injured, 1800 uninjured) following the collision of a cruise ship and an oil tanker close to the Swedish coast. Audio and video recordings of behaviors and communications were reviewed for resilient behaviors based on the DARWIN guidelines using the "resilience markers for small teams" framework (Furniss et al., 2011). Results: A total of 121 observed instances of resilient behaviors were found in the material. In 95 cases (79\%) the observed behaviors followed a priori hypothesized connections between resilient strategies and general markers. Certain marker-strategy combinations occurred frequently, such as 18 observations where the strategy "understand crucial assumptions" occurred together with the marker "adapting to expected and unexpected events." Discussion: Resilience has the potential to contribute to a more holistic disaster management approach. The findings that the observations, in general, correspond to the expected relationship between theoretical concretization and contextualization supports the DARWIN effort to operationalize resilience theory. This is a prerequisite for developing observational 
protocols for training and further studies of resilient behaviors in disaster management teams.

\section{References:}

1. Furniss D., Back J., Blandford A., Hildebrandt M., Broberg H. A resilience markers framework for small teams. Reliab Eng Syst Saf. 2011;96(1):2-10.

Prehosp Disaster Med 2019;34(Suppl. 1):s164-s165 doi:10.1017/S1049023X19003741

\section{Revised Hospital-MIMMS Course for Japan} Dr. Arito Kaji ${ }^{1}$, Dr. Hiromasa Yamamoto ${ }^{2}$, Dr. Naoto Morimura ${ }^{3}$ 1. Emergency and Critical Care Medical Centre, Kishiwada Tokushukai Hospital, Kishiwada, Japan

2. Department of Traumatology and Critical Care Medicine, Osaka City University, Abeno, Japan

3. Department of Acute Medicine, Tokyo University, Bunkyo, Japan

Introduction: HMIMMS (Major Incident Medical Management and Support: The Practical Approach in the Hospital) has been introduced by ALSG (Advanced Life Support Group, Manchester, UK) and developed for many countries for preparing to accept huge numbers of casualties at a hospital during major incidents. The original HMIMMS course has been held in Japan since 2007, produced over 1,200 providers. Japan has a crucial history of natural disasters, earthquakes, tsunamis, and typhoons often resulting in extensive damages to infrastructure and communications.

Aim: The MIMMS-JAPAN and the Japanese Association for Disaster Medicine have joined to plan to revise the original HMIMMS course from the point of view of the difference of the type of disaster.

Method: By the permission of ALSG, two subjects were added "Hospital Evacuation" and "Business Continuity Plan" as lectures, workshops, and tabletops to the original HMIMMS course. Before attending the course, students were required to watch e-learning for deeper understanding and time-saving. Total program was organized into two days.

Results: Main points of modification are to:

1. Replace a system peculiar to the UK with a Japanese system.

2. Add unique contents of a Japanese disaster.

3. Add the important subjects especially in Japan.

4. Modify the presentation slides to understand easily for Japanese students. But the fundamental concept that hospital functions upon 'CSCATTT' is strictly preserved.

Discussion: Newly revised HMIMMS course will start in 2019 for Japanese learners. Many reflections must be accumulated and further revisions will continue.

Prehosp Disaster Med 2019;34(Suppl. 1):s165

doi:10.1017/S1049023X19003753

Risk Mapping of Road Traffic Incidents in Greater Kampala Metropolitan Area for Planning of Emergency Medical Services

Dr. Joseph Kimuli Balikuddembe

Institute for Disaster Management and Reconstruction, Sichuan University, China, and Hong Kong Polytechnic University, Chengdu, China
Introduction: Compared to high-income countries, low and middle-income countries (LMICs) bear the heaviest brunt of road traffic incidents (RTIs), which is a serious public health and development burden. Like other LMICs, Uganda has been experiencing a worryingly high burden of RTIs and their associated impacts with the highest number of all the total registered RTIs in Uganda registered in the Greater Kampala Metropolitan Area (GKMA). This places a tremendous demand on the few existing emergency medical services (EMS) to adequately respond to those affected. Aim: To aid in better planning of EMS for the victims of RTIs by using risk mapping of RTIs in the GKMA.

Methods: A mixed methodological approach involving a systematic review, Delphi panel technique, retrospective data analysis, and a cross-sectional method.

Results: With Uganda progressing forward as envisaged in its "Vision 2040," the GKMA, which is the country's political and socioeconomic epicenter, is experiencing significant changes in terms of population growth. This has significantly increased RTIs, which puts pressure on the pre-hospital emergency care for those affected unless necessary actions are taken.

Discussion: Therefore, the road safety vis-à-vis injury prevention measures, which are needed to reduce the burden of RTIs, should be multifaceted in nature so that they closely correlate with the ongoing dynamics that cause them, particularly in the GKMA which experiences the highest number of RTIs and Uganda as a whole. The WHO "Safe System Approach" is desirable for this purpose as it represents the most appropriate approach because it is broad enough to comprehensively manage any of the ongoing dynamics (political, socio-cultural or economical) that are known to contribute to RTIs.

Prehosp Disaster Med 2019;34(Suppl. 1):s165

doi:10.1017/S1049023X19003765

The Role of the Nurse Disaster Preparedness Coordinator at a Large Suburban Teaching Hospital

Ms. Beth Weeks

University of Colorado Hospital - UCHealth, Denver, United States

Introduction: Mass casualty incidents, whether man-made or natural, are occurring with increasing frequency and severity. Hospitals and health systems across the United States are striving to be more rigorously prepared more such incidents. Following a mass shooting in 2012 and significant growth and expansion of our hospital and health system in the following years, a need was identified for more staff to support preparedness efforts.

Aim: To discuss the roles and responsibilities of Nurse Disaster Preparedness Coordinator (NDPC), a dedicated position in the Emergency Department (ED).

Methods: The role of Nurse Disaster Preparedness Coordinator was implemented in 2016, is a part-time position in the Emergency Department and reports to the ED Manager while working closely with the ED Director of Emergency Preparedness and the hospital Emergency Manager. The role addresses all areas of the emergency 